\title{
Risk Factors For Nosocomial Pneumonia: Comparing Adult Critical-Care Populations
}

\author{
KENJI M. CUNNION, DAVID J. WEBER, W. EUGENE BROADHEAD, LAURA C. HANSON, \\ CARL F. PIEPER, and WILLIAM A. RUTALA
}

School of Public Health, University of North Carolina; Divisions of Infectious Diseases, General Medicine and Hospital Epidemiology, University of North Carolina Hospitals, Chapel Hill, North Carolina; and Research and Education and Biometry and Medical Informatics Divisions of the Department of Community and Family Medicine, Duke University Medical Center, Durham, North Carolina

\begin{abstract}
The purpose of the study was to examine risk factors for nosocomial pneumonia in the surgical and medical/respiratory intensive care unit (ICU) populations. In a public teaching hospital, all cases of nosocomial pneumonia in the surgical and medical/respiratory ICUs $(n=20$, respectively) were identified by prospective surveillance during a 5-yr period from 1987-1991. Each group of ICU cases was compared with 40 ICU control patients who did not acquire pneumonia, and analyzed for 25 potential risk factors. Surgical ICU patients were found to have consistently higher rates of nosocomial pneumonia than medical ICU patients ( $R R=2.2$ ). The strongest predictor for nosocomial pneumonia in both the surgical and medical/respiratory ICU groups was found to be prolonged mechanical ventilation $(>1 \mathrm{~d})$ resulting in a 12-fold increase in risk over nonventilated patients. APACHE III score was found to be predictive of nosocomial pneumonia in the surgical ICU population, but not in the medical/respiratory ICU population. We conclude that certain groups deserve special attention for infection control intervention. Surgical ICU patients with high APACHE scores and receiving prolonged mechanical ventilation may be at the greatest risk of acquiring nosocomial pneumonia of all hospitalized patients. Cunnion KM, Weber DI, Broadhead WE, Hanson LC, Pieper CF, Rutala WA. Risk factors for nosocomial pneumonia: comparing adult critical-care populations.
\end{abstract}

AM J RESPIR CRIT CARE MED 1996;153:158-62.

Pneumonia is the second most common type of nosocomial infection in the United States, and carries the highest mortality rate of any nosocomial infection (1). Most epidemiologic studies of such infection have found overall hospital nosocomial pneumonia rates per admission to range from 0.66 to $3 \%$. This rate of infection is sharply higher for critical care patients, in whom pneumonia complicates from 8 to $22 \%$ of intensive care unit (ICU) admissions (2). The mortality rate in patients with nosocomial pneumonia has ranged from 20 to $50 \%$ (3). Nosocomial pneumonia was reported in one study to be associated with $15.5 \%$ of all hospital deaths, some of which may be preventable (4). The cost for diagnosing and treating nosocomial pneumonia has been estimated at $\$ 2$ billion annually (5).

The pathogenesis of nosocomial pneumonia begins with colonization of the oropharyngeal mucosa of a susceptible host. Because of factors that impair respiratory defenses, the host then aspirates pathogenic bacteria (2). Multivariate studies have shown that the major risk factors associated with nosocomial pneumonia include mechanical ventilation, severity of illness, neurologic impairment, depressed consciousness, immunosuppresion, poor nutritional status, chronic lung disease, thoracoabdominal surgery, and observed high-volume pulmonary aspiration (6-9).

(Received in original form May 14, 1993 and in revised form February 3, 1995) Correspondence and requests for reprints should be addressed to Kenji $M$. Cunnion, 2543 Potomac Hunt Ln. \#2A, Richmond, VA 23233.

Am J Respir Crit Care Med Vol 153. pp 158-162, 1996
Prior studies of nosocomial pneumonia have not examined differences between surgical and medical ICU populations, although some accepted risk factors, such as thoracoabdominal surgery, clearly affect surgical patients. Clinical observations suggest that surgical and medical ICU patients differ in many ways, and are likely to have different risk factors for nosocomial pneumonia. The purpose of this study was to describe the specific incidences of nosocomial pneumonia among medical and surgical ICU patients, and to examine differences in risk factors for nosocomial pneumonia in the surgical and medical ICU populations.

\section{METHODS}

The study subjects were patients admitted to the ICUs of the University of North Carolina Hospitals (UNCH), a 600-bed teaching hospital, during the period from August 1, 1987 to December 31, 1991. During this 5 -yr period, 3,392 patients were admitted to the surgical ICU and 4,173 patients were admitted to the medical/respiratory ICU.

Cases of nosocomial pneumonia were identified by prospective surveillance throughout the study period. All nosocomial infections at $\mathrm{UNCH}$ are recorded in a data base collected by the Hospital Epidemiology Division since 1978. Trained infection-control nurses are responsible for identifying and confirming cases of nosocomial infection. Cases are detected by review of positive culture reports from the microbiology laboratory. Patients from whom cultures are not obtained are visited by one of the infection-control practitioners to ascertain cross-infection. All autopsy reports are reviewed for possible nosocomial infection. $\mathrm{Pa}$ tients meeting U.S. Centers for Disease Control and Prevention (CDC) criteria for nosocomial pneumonia (10), and having radiologic evidence 
of the disease, are entered in a prospective manner into a computerized epidemiology information retrieval system (11). Data about patients acquiring nosocomial infections are then reviewed monthly by a physician trained in infectious diseases.

For CDC surveillance purposes, nosocomial pneumonia in persons over the age of 12 mo must meet one of the following criteria: (1) rales or dullness to percussion on physical examination of the chest and any of the following: purulent sputum of new onset or a change in character of sputum; a pathogenic organism isolated from blood culture; a pathogen isolated from a specimen obtained by transtracheal aspiration, bronchial brushing, or biopsy; or (2) a chest radiographic examination showing new or progressive infiltration, consolidation, cavitation, or pleural effusion and any of the following: purulent sputum of new onset or a change in character of sputum; isolation of a pathogenic organism from blood culture; isolation of a pathogen from a specimen obtained by transtracheal aspiration, bronchial brushing, or biopsy; isolation of virus or detection of viral antigens in respiratory secretions; a diagnostic single antibody titer $(\operatorname{IgM})$ or a fourfold increase in paired serum samples ( $\operatorname{IgG})$ for a pathogen. UNC Hospitals requires the second of these CDC criteria for the diagnosis of pneumonia (i.e., a chest radiograph showing new or progressive infiltration).

The incidence of nosocomial pneumonia per ICU admission was determined for the surgical (SICU) and for the combined medical/respiratory ICUs (M/RICU) for the years 1987 through 1991. The total number of cases of nosocomial pneumonia for each ICU by year, and the total number of patients admitted to each ICU by year, were obtained from the data base of the hospital's epidemiology information retrieval system.

A case-control study was performed to identify risk factors for nosocomial pneumonia in each unit. Cases were defined as persons acquiring a pneumonia $48 \mathrm{~h}$ or more after admission to an ICU and for up to $48 \mathrm{~h}$ after discharge from an ICU. 、

Cases of nosocomial pneumonia for the M/RICU consisted of all patients contracting such pneumonia in these two units during this interval. Patients with pneumonia at the time of admission to the ICU were excluded. Because the incidence of pneumonia in the SICU was higher, cases for this unit were randomly selected from all patients acquiring a nosocomial pneumonia in the unit during the study period $(n=75)$, such that the total number of SICU cases $(n=20)$ evaluated equaled the total combined number of M/RICU cases evaluated. The sample size from each group was equalized so that each leg of the casecontrol study would have equal statistical power and a meaningful comparison of risk factors could be made.

Many of the cases originally identified as having nosocomial pneumonia in the nosocomial infection data base were later excluded. Of the cases initially identified, $40 \%$ were found to have acquired their pneumonia within $48 \mathrm{~h}$ after admission to the ICU. A further $15 \%$ of the cases were rejected from the study because: $(I)$ the part of the chart containing the pertinent ICU admission was unavailable; or (2) there were insufficient data in the chart from which to determine whether the risk factors analyzed in this study were present. These phenomena were equally predominant in each ICU.

Controls were chosen from admission census logs recorded by each of the individual ICUs. The logs are sorted by date of admission to the ICU. Controls were the first eligible patients admitted to the same ICU following the admission of each of the case patients. This method was used to achieve a representative and relatively random sample of ICU patients. To be an eligible control, a patient had to remain in the ICU for longer than $48 \mathrm{~h}$ and not acquire a pneumonia while in the unit. Patients admitted with a diagnosis of pneumonia were also excluded. Controls were matched with the cases in a 2:1 ratio. Additional controls were not reviewed, since no more statistical power would have been gained from their use.

For the purposes of this study, no case or control was used more than once, regardless of whether or not they acquired another nosocomial pneumonia at a different time or had another admission to an ICU where no pneumonia was acquired (making the patient a potential control).

Once the cases and controls were identified, data on demographics and on potential risk factors were determined by chart review. All chart reviews were conducted by one of the authors (K.M.C.). Only objective and routinely collected data were studied, and only risk factors preced- ing the onset of infection were recorded. Operational definitions were used to ensure repeatability of measurements.

Demographic data and method of payment, a surrogate measure of socioeconomic status, were recorded. Risk factors acquired prior to admission included poor nutrition (initiation of hyperalimentation through intravenous or nasogastric nutritional supplementation), a history of smoking, chronic obstructive pulmonary disease (COPD), chronic liver disease (prothrombin time $>15 \mathrm{~s}$ ), neuromuscular disease, chronic cognitive impairment, diabetes mellitus, malignancy, prior infection, and current use of immunosuppressive medication.

Potential risk factors acquired during the hospitalization included high-volume pulmonary aspiration; depressed level of consciousness (Glasgow Coma Score [GCS]); surgical procedures; mechanical ventilation prior to pneumonia; nasogastric intubation; and use of an antacid, histamine antagonist, immunosuppressive antibiotic, or bronchodilator medication. The medications were recorded only if taken for at least 7 of the $30 \mathrm{~d}$ preceding infection. Days at risk was defined as days in the ICU prior to the onset of pneumonia (for cases) or discharge (for controls). Severity of illness was assessed by the acute physiology and chronic health evaluation (APACHE) III scoring system (12), using the worst values recorded during the $48 \mathrm{~h}$ prior to the diagnosis of pneumonia. This window was chosen as the most likely to represent the period when nosocomial pneumonia was established.

APACHE III is based on the worst data parameters observed over a 24-h period. A 48-h window was chosen for this study, in order to allow more complete filling of all data parameters. No more than two of 20 parameters were absent for any patient. APACHE III allows for assigning null values to incomplete parameters, permitting a reasonably accurate score to be calculated for all patients.

Risk factors for acute conditions (e.g., low albumin, hyperalimentation, etc.) were counted as positive for controls if the condition existed during the $7 \mathrm{~d}$ prior to ICU admission or during the course of the ICU stay. Mechanical ventilation was recorded for consecutive days prior to discharge from the ICU. For controls, the APACHE III score and GCS were calculated from the worst values recorded during the first $48 \mathrm{~h}$ after admission to the ICU.

Outcome measures investigated in this study were length of stay after admission to the ICU and death during admission. Culture results for patients acquiring nosocomial pneumonia were also recorded. A dominant organism detected by Gram stain in the first culture after diagnosis was taken as the causative organism.

\section{Statistical Analysis}

Univariate analyses were performed for potential risk factors in order to identify those statistically associated with nosocomial pneumonia. Three analyses were performed in parallel, one comparing SICU cases with SICU controls, one comparing MICU and RICU cases with their controls, and one comparing all cases (MICU, RICU, and SICU) with all controls. Chi-squared statistics were calculated for dichotomous variables. Continuous variables that were normally distributed were compared using the Student's $t$ test. Non-normally-distributed continuous variables were compared using Wilcoxon's ranked sign test. Comparisons were unpaired, and all tests of significance were two-tailed.

Univariate analyses were performed on 25 potential risk factors: hyperalimentation, smoking, COPD, chronic liver disease, end-stage renal disease, immunocompromising disease, neuromuscular disease, chronic cognitive impairment, diabetes mellitus, malignancy, prior infection, immunosuppressive medications, high-volume pulmonary aspiration, recent surgery, endotracheal intubation, nasogastric intubation, antacids, histamine antagonists, immunosuppressive antibiotics, bronchodilators, chemotherapy, shock, GCS score, and APACHE III score. Variables yielding an alpha value of 0.05 or less were included in the multiple logistic regression model. GCS and APACHE III scores were also included in multivariate analyses as measures of decreased mental status and severity of illness.

An initial multiple logistic regression included all variables in the model. In order to determine independent risk factors for nosocomial pneumonia, a backward stepwise elimination was performed on this model, with only those variables attaining an alpha value of 0.05 being retained. The models were run twice, once with mechanical ventilation defined as present or absent and a second time with positive mechanical 


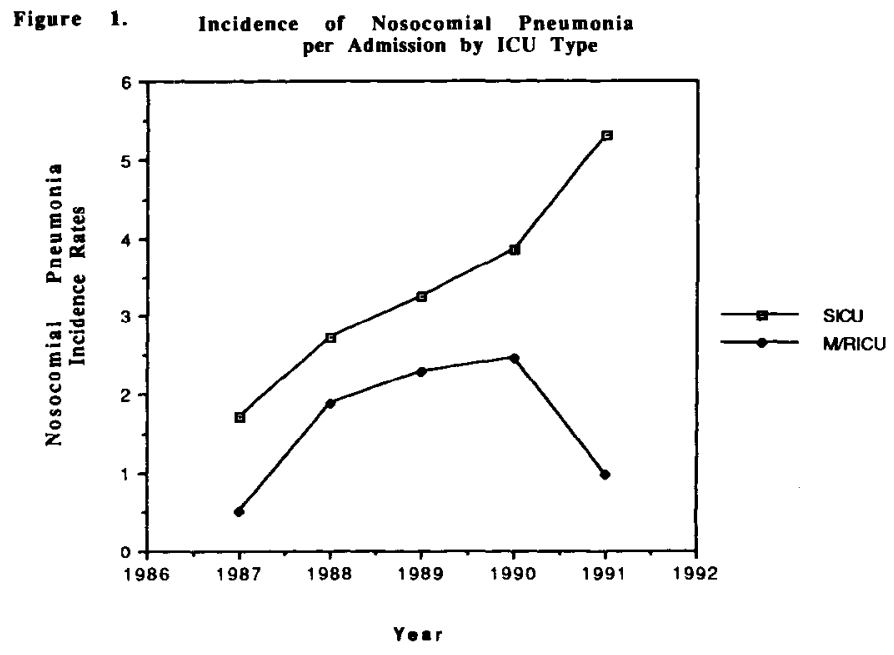

Figure 1. Incidence of nosocomial pneumonia (\%) per admission by ICU type.

ventilation defined as mechanical ventilation for $2 \mathrm{~d}$ or more. Stated odds ratios were derived from the multiple logistic regression.

\section{RESULTS}

\section{Time Trends}

As hypothesized, the rates of infection differed markedly for SICU and M/RICU patients. Data on the incidence of nosocomial pneumonia per admission for each ICU by year showed the SICU to have a consistently higher incidence of nosocomial pneumonia than the M/RICU for all years studied (Figure 1). The incidence of nosocomial pneumonia for the SICU also increased more than threefold over the 4-yr study period, from 1.7 to $5.3 \%$ of admissions.

\section{Univariate Analysis}

Univariate analyses was performed to determine potential risk factors associated with nosocomial pneumonia. No significant associations were found in univariate comparisons of cases with nosocomial pneumonia and controls when analyzing age, gender, ethnic status, or method of payment (Table 1). Cases of nosocomial pneumonia had one more day at risk on average than did controls, but the differences were not significant. Demographic variables were not entered into multiple regression models.

Several patient and hospital treatment factors differed significantly for cases and controls (Table 2). Mechanical ventilation lasting two or more days was found to be strongly associated

TABLE 1 CHARACTERISTICS OF THE STUDY POPULATION

\begin{tabular}{llll} 
& Status & SICU & M/RICU \\
\hline Age, yr & Case & 57.90 & 52.45 \\
Male, \% & Control & 53.35 & 58.68 \\
Nonwhite, \% & Case & 70.0 & 55.0 \\
Public or self pay, \% & Control & 60.0 & 57.0 \\
& Case & 25.0 & 25.0 \\
Days at risk & Control & 40.0 & 40.0 \\
& Case & 25.0 & 30.0 \\
& Control & 30.0 & 27.5 \\
& Case & 6.15 & 7.55 \\
\hline
\end{tabular}

$n=20$ for SICU cases and M/RICU cases.

$n=40$ for SICU controls and $M / R I C U$ controls.
TABLE 2

\begin{tabular}{|c|c|c|c|c|}
\hline & Status & SICU & M/RICU & All \\
\hline \multirow{2}{*}{$\begin{array}{l}\text { Mechanical ventilation, } \% \\
\qquad(\geqslant 1 \text { d) }\end{array}$} & Case & 95.0 & $95.0^{\dagger}$ & $95.0^{\dagger}$ \\
\hline & Control & 82.5 & 50.0 & 66.3 \\
\hline \multirow{2}{*}{$\begin{array}{l}\text { Mechanical ventilation, } \% \\
(\geqslant 2 \mathrm{~d})\end{array}$} & Case & $95.0^{\star}$ & $90.0^{\dagger}$ & $92.5^{\dagger}$ \\
\hline & Control & 57.5 & 40.0 & 48.8 \\
\hline \multirow[t]{2}{*}{ Glasgow Coma Scale score** } & Case & 12.0 & $10.9^{\star}$ & $11.4^{\star}$ \\
\hline & Control & 13.4 & 13.0 & 13.2 \\
\hline \multirow[t]{2}{*}{ APACHE III score $* *$} & Case & $62.4^{\star}$ & 68.3 & $65.3^{\star}$ \\
\hline & Control & 47.6 & 59.1 & 53.4 \\
\hline \multirow[t]{2}{*}{ Nasogastric intubation, $\%$} & Case & 95.0 & $90.0^{\dagger}$ & $92.5^{*}$ \\
\hline & Control & 87.5 & 50.0 & 68.8 \\
\hline \multirow[t]{2}{*}{ Hyperalimentation, \% } & Case & $65.0^{\star}$ & $55.0^{*}$ & $60.0^{\dagger}$ \\
\hline & Control & 32.5 & 27.5 & 30.0 \\
\hline
\end{tabular}

${ }^{*} p \leqslant 0.05$.

$\dagger p \leqslant 0.002$.

$n=20$ for SICU cases and M/RICU cases.

$n=40$ for SICU controls and M/RICU controls.

$n=40$ for ALL cases; $\Pi=80$ for ALL controls.

** Glasgow Coma Scale score and APACHE III score are expressed as averages.

with nosocomial pneumonia for each ICU group. When mechanical ventilation was defined as present or absent it was strongly associated with nosocomial pneumonia for the M/RICU population but not for the SICU population. For SICU patients, no greater risk of pneumonia was found for patients receiving only one day of mechanical ventilation than for those receiving no mechanical ventilation.

High APACHE III scores, indicating severe illness, showed a strong tendency to predict nosocomial pneumonia for SICU patients but not for M/RICU patients. There was also a tendency for low GCS scores, which indicate more severe neurologic impairment, to be associated with nosocomial pneumonia for both SICU and RICU patients.

The use of hyperalimentation (a surrogate measure for poor nutritional status) tended to predict nosocomial pneumonia for both SICU and M/RICU patients. The use of nasogastric intubation was found to be significantly associated with nosocomial pneumonia in M/RICU patients but not in SICU patients. Strong associations were also found between nosocomial pneumonia and both chronic liver disease and high-volume pulmonary aspiration when all subjects were considered, but these variables were not significant in the analysis of M/RICU or SICU patients separately. They were not entered into the multivariate analysis because of the small numbers $(n=5)$ of patients with these potential risk factors. No significant associations were found between the other potential risk factors and nosocomial pneumonia.

\section{Multivariate Analysis}

Multiple logistic regression was performed to determine independent predictors for nosocomial pneumonia (Table 3). The model for the SICU patients included mechanical ventilation, APACHE III score, and hyperalimentation. After a backward stepwise elimination was performed on the model for the SICU, mechanical ventilation ( $\geqslant 2 \mathrm{~d}$ ) and APACHE score were found to be significantly associated with nosocomial pneumonia.

The multiple logistic regression model for the M/RICU patients included mechanical ventilation, GCS score, hyperalimentation, and nasogastric intubation. After a backward stepwise elimination was performed on the $\mathrm{M} / \mathrm{RICU}$ model, only mechanical ventilation $(\geqslant 2 \mathrm{~d})$ remained significant.

The regression model for all subjects included mechanical ventilation, APACHE III score, GCS score, hyperalimentation, and 
TABLE 3

MULTIVARIATE ANALYSIS: INDEPENDENT PREDICTORS OF NOSOCOMIAL PNEUMONIA*

\begin{tabular}{llcr}
\hline & Significant Predictors & Odds Ratio & p Value \\
\hline SICU & Mechanical ventilation & 11.6 & 0.03 \\
& APACHE III score & $1.9^{\dagger}$ & 0.05 \\
M/RICU & Mechanical ventilation & 13.5 & $<0.01$ \\
All & Mechanical ventilation & 12.1 & $<0.01$ \\
& APACHE III score & $1.6^{\dagger}$ & 0.04 \\
\hline
\end{tabular}

* Multiple logistic regression with a backward stepwise elimination, alpha $=0.05$

$\dagger$ Odds ratio for a 20-point change in APACHE III score.

nasogastric intubation. Backward stepwise elimination revealed mechanical ventilation ( $\geqslant 2 \mathrm{~d}$ ) and APACHE III score to be significant predictors of nosocomial pneumonia.

\section{Outcome Analysis}

Outcome variables were described for SICU and M/RICU cases (Table 4). Mean length of hospitalization after admission to the ICU was greater for cases of nosocomial pneumonia in each ICU group. M/RICU cases tended to have longer average lengths of stay than did SICU cases $(p=N S)$. Death during hospitalization was significantly associated with nosocomial pneumonia in both ICU categories $(\mathrm{p}<0.001)$. The acquisition of nosocomial pneumonia resulted in a 23-fold increase in risk of death in the SICU and a 15-fold increase in the M/RICU. SICU cases were slightly more likely than M/RICU cases to have pneumonia at the time of death $(p=N S)$.

Culture results were obtained for 39 of 40 cases of nosocomial pneumonia, in nine $\mathbf{2 3 . 1 \%}$ ) of which no bacterial growth or normal flora were found. In the remaining 30 cases, 37 organisms were identified. The distribution of cultured organisms was similar in the two ICU populations (Table 5). A greater incidence of nosocomial pneumonia due to Staphylococcus aureus and Candida was found in the M/RICU than in the SICU population.

\section{DISCUSSION}

The purpose of this study was to examine differences in incidence rates and risks factors for nosocomial pneumonia in different adult critical-care populations in a teaching hospital. The major findings of this study confirm the importance of investigating these populations separately. SICU patients were found to be at greater risk of acquiring nosocomial pneumonia than were M/RICU patients, and this risk difference appears to be increasing each year. The APACHE III score was found to be a good predictor for nosocomial pneumonia in the SICU but not in the M/RICU population. Finally, although mechanical ventilation alone increased the risk of nosocomial pneumonia in the M/RICU population, the duration of mechanical ventilation was what predicted the risk of nosocomial pneumonia in the SICU population.

Four major reported studies have investigated multiple risk factors for nosocomial pneumonia (6-9). Of these, only one was limited to critical-care patients (6). All of these studies combined different critical-care populations and considered them as a single homogeneous group. The SICU and M/RICU populations are generally considered to differ in their clinical illnesses and the care they receive, but our study is the first to examine how these differences may influence these patients' risk of acquiring nosocomial pneumonia.

SICU patients were found to be at greatest risk of acquiring pneumonia, and to have an increasing risk for this. This is a worrisome trend deserving increased attention from infection-control practitioners as well as caregivers in the SICU. The low rate of infection in the study ICUs relative to rates reported in the literature suggests that this phenomenon does not reflect a problem unique to our institution. No data are available in the literature against which to compare our findings and evaluate whether they are consistent with trends elsewhere, but we do feel that further investigation of trends in ICU-acquired pneumonia is warranted.

The cause of this increasing risk of infection may be more frequent surgery for patients with severe chronic illness than was done in the past. Other possible explanations include an increasing number of organ-transplant patients and improved shortterm survival for patients with massive acute trauma. As surgeons become more willing to operate on increasingly debilitated patients, the SICU population may manifest more postoperative infection.

Duration of mechanical ventilation strongly influenced the risk of nosocomial pneumonia in the SICU population. Patients intubated only during general anesthesia were not at greater risk of nosocomial pneumonia than surgical patients never intubated. However, those patients requiring prolonged mechanical ventilation in the SICU have a 12-fold greater risk of acquiring nosocomial pneumonia than do all other SICU patients. Extra care should be taken to prevent pneumonia among patients receiving prolonged ventilation. Such care includes meticulous handwashing and ensuring that condensate in the ventilation tubing, a recognized breeding ground for pathogenic bacteria, does not drain into the patient's lungs when the tubing is changed or moved. The use of antacids and histamine antagonists should be carefully evaluated for each patient, since alkalization of the stomach is known to result in colonization with bacteria that may then be aspirated. Nonabsorbable p.o. antibiotics taken eternally may in the future be useful for decreasing the risk of nosoco-

TABLE 4

MORBIDITY AND MORTALITY ASSOCIATED WITH NOSOCOMIAL PNEUMONIA

\begin{tabular}{|c|c|c|c|c|c|}
\hline & Status & SICU & Odds Ratio & M/RICU & Odds Ratio \\
\hline \multicolumn{6}{|l|}{ Length of stay } \\
\hline \multirow[t]{2}{*}{ (following ICU admission) } & Case & 30.0 & & 40.9 & \\
\hline & Control & 22.3 & & 23.1 & \\
\hline \multirow[t]{2}{*}{ Death, $\%$} & Case & 55.0 & $23.2^{\star}$ & 55.0 & $15.1^{*}$ \\
\hline & Control & 05.0 & & 07.5 & \\
\hline \multicolumn{6}{|l|}{ Nosocomial pneumonia } \\
\hline present at time of death, $\%$ & Died & 81.8 & & 63.6 & \\
\hline \multicolumn{6}{|c|}{${ }^{*} p \leqslant 0.002$} \\
\hline $\begin{array}{l}\text { Length of stay in days. } \\
n=20 \text { for SICU cases and } M / R \\
n=40 \text { for SICU controls and } \\
n=11 \text { for SICU cases with dea } \\
n=11 \text { for } M / R I C U \text { cases with }\end{array}$ & $\begin{array}{l}\text { es. } \\
\text { controls. } \\
\text { g hospita } \\
\text { uring hosp }\end{array}$ & & & & \\
\hline
\end{tabular}


TABLE 5

CULTURES OF CAUSATIVE ORGANISMS*

\begin{tabular}{llcc}
\hline & \multicolumn{1}{c}{ Organism } & SICU & M/RICU \\
\hline Culture Results & Pseudomonas aeruginosa & 27.8 & 15.8 \\
& Staphylococcus aureus & 11.1 & 21.1 \\
& Klebsiella pneumoniae & 0 & 15.8 \\
& Hemophilus influenzae & 11.1 & 5.3 \\
& Candida ssp. & 0 & 10.5 \\
\hline
\end{tabular}

* Results are expressed as percentages.

mial pneumonia. Several small studies have shown that selective decontamination of the oropharynx and gastrointestinal tract decreases the risk of nosocomial pneumonia (13).

The APACHE III score was found to predict nosocomial pneumonia in the SICU but not in the M/RICU population. There was a wider disparity in severity of illness between cases and controls in the SICU than in the M/RICU (Table 2). This was probably due a greater heterogeneity in the severity of illness in the SICU population. This difference in severity of illness in SICU patients is detectable in their APACHE III scores, and is important in identifying those patients at increased risk for pneumonia. $\mathrm{M} / \mathrm{RICU}$ patients appear to be much more homogeneous in terms of their burden of illness, and the APACHE III score is not useful in identifying $\mathrm{M} / \mathrm{RICU}$ patients at increased risk for pneumonia. A marker for increased risk of nosocomial pneumonia in one unit cannot be blindly applied to all critical-care populations with the assumption that it will also be predictive for these populations.

When all subjects in this study are considered, both mechanical ventilation and the APACHE III score were significantly associated with nosocomial pneumonia. This would tend to indicate that the APACHE III score is a good predictor for pneumonia in all adult ICU patients. However, when the units are considered separately, this proves not to be the case, with the APACHE III score a strong predictor of pneumonia only in the SICU population. This emphasizes the importance of considering these populations separately.

Death was the outcome of nosocomial pneumonia in the majority of patients in both ICU groups $(55 \%)$. However, nosocomial pneumonia was found to be more often directly causative of death in the SICU population $(82 \%)$ than in the M/RICU population $(64 \%)$. The low mortality rate among controls reflects the occurrence of most deaths not related to nosocomial pneumonia within the first $48 \mathrm{~h}$ of ICU stay. Nosocomial pneumonia is a major cause of mortality in M/RICU patients, but M/RICU patients acquiring nosocomial pneumonia are more likely to recover from such pneumonia prior to death from other causes. $S$. aureus and Candida were more likely to be causative organisms of nosocomial pneumonia in the $\mathrm{M} / \mathrm{RICU}$ population than in the SICU population.

Possible limitations of this study should be considered when interpreting its results. Although the study cases were collected over a 5-yr period, the small number of cases in the M/RICU population limited the statistical power of the study. Cases were derived from patients at a single hospital, but the risk factors found to be associated with nosocomial pneumonia in this study are consistent with those cited in the literature. The study was retrospective and could only examine potential risk factors in the medical record, but operational definitions were used to ensure the accuracy of those factors that were examined.
Future studies of nosocomial pneumonia in critical-care populations should make allowances so that different ICUs may be considered separately, in order to avoid false generalizations. Care should also be taken to consider the duration of mechanical ventilation and its association with an increased risk of nosocomial pneumonia. Several potential risk factors, such as the GCS score, nasogastric intubation, and hyperalimentation deserve further study, since they showed themselves to be strong, but not significant, independent predictors of nosocomial pneumonia in this study.

The major implication of these results is that certain groups deserve special attention for infection-control intervention. SICU patients with high APACHE III scores and more than one day of mechanical ventilation are perhaps at the greatest risk among all hospitalized patients of acquiring nosocomial pneumonia. SICU patients are more likely than M/RICU patients to die as a direct consequence of this complication. If effective preventive measures against nosocomial pneumonia can be designed, this group of patients will derive important benefits from them.

\section{References}

1. Craven, D. E., and K. A. Stegner. 1989. Nosocomial pneumonia in the intubated patient. Infect. Dis. Clin. North Am. 3:843-866.

2. Craven, D. E., T. W. Barber, K. A. Stenger, and M. A. Montecalvo. 1990. Nosocomial pneumonia in the 1990s: update of the epidemiology and risk factors. Semin. Respir. Infect. 5:157-172.

3. Gross, P. A. 1987. Epidemiology of hospital acquired pneumonia. Semin. Respir. Infect. 2:2-7.

4. Gross, P. A., H. C. Neu, and P. Aswapokee. 1980. Deaths from nosocomial infections: experience in a university hospital and a community hospital. Am. J. Med. 68:218-223.

5. Wenzel, R.P. 1980. Hospital-acquired pneumonia: overview of the current state of the art prevention and control. Eur. J. Clin. Microbiol. Infect. Dis. 8:56-60.

6. Craven, D. E., L. M. Kunches, V. Kilinsky, D. A. Lichtenberg, B. J. Make, and W. R. McCabe. 1986. Risk factors for nosocomial pneumonia and fatality in patients receiving continuous mechanical ventilation. Am. Rev. Respir. Dis. 133:792-796.

7. Celis, R., A. Torres, J. M. Gatell, M. Almela, R. Rodrriguez-Roisin, and A. Agusti-Vidal. 1988. Nosocomial pneumonia: a multivariate analysis of risk and prognosis. Chest 93:318-324.

8. Hanson, L. C., D. J. Weber, W. A. Rutala, and G. P. Samsa. 1992. Risk factors for nosocomial pneumonia in the elderly. Am. J. Med. 92:161-166.

9. Hooten, T. M., R. W. Haley, D. H. Culver, J. W. White, W. M. Morgan, and R. J. Carroll. 1981. The joint association of multiple risk factors with the occurrence of nosocomial infection. Am.J. Med. 70:960-970.

10. Garner, J. S., W. R. Jarvis, T. G. Emori, T. C. Horan, and J. M. Hughes. 1988. CDC definitions for nosocomial infection, 1988. Am. J. Infect. Control 16:128-140.

11. Brawley, R. L., D. J. Weber, G. P. Samsa, and W. A. Rutala. 1989. Multiple nosocomial infections: an incidence study. Am. J. Epidemiol. 130:769-780.

12. Knaus, W. A., D. P. Wagner, E. A. Draper, J. E. Zimmerman, M. Bergner, P. G. Bestos, C. A. Sirio, D. J. Murphy, T. Lotring, A. Damiano, and F. E. Harrell. 1991. The APACHE III prognostic system: risk prediction of hospital mortality in critically ill hospitalized adults. Chest 100:1619-1636.

13. Flaherty, J. P., and R. A. Weinstein. 1990. Infection Control and Pneumonia Prophylaxis Strategies in the Intensive Care Unit. Semin. Respir. Infect. 5:191-203. 